\title{
Engaging policy makers in road safety research in Malaysia: a theoretical and contextual analysis
}

\begin{abstract}
Road traffic injuries (RTIs) are a growing public health problem that must be addressed through evidence-based interventions including policy-level changes such as the enactment of legislation to mandate specific behaviors and practices. Policy makers need to be engaged in road safety research to ensure that road safety policies are grounded in scientific evidence. This paper examines the strategies used to engage policy makers and other stakeholder groups and discusses the challenges that result from a multi-disciplinary, inter-sectoral collaboration. A framework for engaging policy makers in research was developed and applied to describe an example of collective road safety research in Malaysia. Key components of this framework include readiness, assessment, planning, implementation/evaluation, and policy development/sustainability. The case study of a collaborative intervention trial for the prevention of motorcycle crashes and deaths in Malaysia serves as a model for policy engagement by road safety and injury researchers. The analytic description of this research process in Malaysia demonstrates that the framework, through its five stages, can be used as a tool to guide the integration of needed research evidence into policy for road safety and injury prevention.
\end{abstract}

Keyword: Evidenced-based decision-making; Policy engagement; Collective research; Knowledge translation; Knowledge brokering; Malaysia; Road safety; Policy framework; Engaging policy makers in road safety 